\title{
Physical activity in pregnancy prevents gestational diabetes: A meta-analysis
}

\author{
Suhail A.R. Doi ${ }^{a, *}$, Luis Furuya-Kanamori ${ }^{a, b}$, Egon Toft ${ }^{c}$, Omran A.H. Musa ${ }^{a}$, \\ Aisha M. Mohamed ${ }^{a}$, Justin Clark ${ }^{d}$, Lukman Thalib ${ }^{e}$ \\ ${ }^{a}$ Department of Population Medicine, College of Medicine, QU Health, Qatar University, Doha, Qatar \\ ${ }^{\mathrm{b}}$ Research School of Population Health, Australian National University, Canberra, Australia \\ ${ }^{\mathrm{c}}$ Deans Office, College of Medicine, QU Health, Qatar University, Doha, Qatar \\ ${ }^{\mathrm{d}}$ The Centre for Research into Evidence Based Practice, Bond University, Gold Coast, Australia \\ ${ }^{\mathrm{e}}$ Department of Public Health, College of Health Sciences, QU Health, Qatar University, Doha, Qatar
}

\section{A R T I C L E I N F O}

Article history:

Received 10 April 2020

Received in revised form

15 July 2020

Accepted 10 August 2020

Available online 20 August 2020

Keywords:

Gestational diabetes mellitus

Exercise

Obesity

Overweight

Pregnancy risk

\begin{abstract}
A B S T R A C T
Aims: The effectiveness of physical activity (PA) programs for prevention of gestational diabetes (GDM) lacks conclusive evidence. The aim of this study was to generate clear evidence regarding the effectiveness of physical activity programs in GDM prevention to guide clinical practice.

Methods: PubMed/Medline, ISI Web of Science, Scopus, and EMBASE were searched to identify the randomized trials (RCTs) published until June 2019. Randomised controlled trials enrolling women at high risk before the 20th week of gestation comparing the effect of PA interventions with usual care for prevention of GDM were retrieved. Data obtained were synthesised using a bias-adjusted model of meta-analysis.

Results: A total of 1467 adult women in 11 eligible trials were included. The risk of GDM was significantly lower with PA, but only when it was delivered in the healthcare facility (RR 0.53; 95\% CI 0.38-0.74). The number needed to treat with PA in pregnancy (compared to usual care) to prevent one GDM event was 18 (95\% CI $14-29)$. The overall effect of PA interventions regardless of location of the intervention was RR 0.69 (95\% CI $0.51-0.94$ ).

Conclusions: This study provides evidence that in-facility physical activity programs started before the 20th week of gestation can significantly decrease the incidence of GDM among women at high risk.
\end{abstract}

(c) 2020 Elsevier B.V. All rights reserved.

\section{Introduction}

With the current obesity epidemic, gestational diabetes mellitus (GDM) has also been on the rise given that the key risk fac- tor is maternal adiposity [1]. GDM is also associated with women of an advanced age at pregnancy, having a family history of diabetes or a past history of GDM or macrosomia [2-5]. Development of GDM has implications for both the mother

\footnotetext{
* Corresponding author at: Department of Population Medicine \& Endocrine Specialist, College of Medicine, Qatar University, Doha, Qatar.

E-mail addresses: sardoi@gmx.net, sdoi@qu.edu.qa (S.A.R. Doi). 
(e.g. interventions to manage hyperglycemia) and the baby (e.g. birth complications or longer-term dysmetabolic traits in the child) [6].

Preventing GDM is a priority in pregnancy. Several interventions to mitigate hyperglycemia and avoid birth complications (e.g. macrosomia) have been suggested, the main ones being pharmacological, physical activity and lifestyle counselling [7]. Results with metformin have been disappointing, with the latest synthesis of randomised controlled trials (RCT's) conclusively demonstrating a lack of efficacy in preventing GDM [8]. Nutrition counseling, diets, physical activity (PA) programs and PA counselling have also been extensively trialed in pregnancy. The rationale for the latter is that GDM is linked to insulin resistance on a backdrop of beta-cell dysfunction [9] and such interventions modulate insulin sensitivity due to their possible effect on gestational weight gain [7]. Evidence has been generated since 2002, when the first RCT of counseling (nutrition and/or physical activity) in a high risk population was attempted [10]. Nonetheless, recent multicentre RCTs including the LIMIT [11], UK Pregnancies Better Eating and Activity (UPBEAT) [12], Finnish Gestational Diabetes Prevention (RADIEL) [13] and the Vitamin D and Lifestyle Intervention (DALI) [14] trials that evaluated the effect of counselling among pregnant women at high risk have all been disappointing in terms of GDM prevention. What is unclear is if direct PA programs as the intervention (as opposed to counselling) can make a difference.

There have also been many attempts to synthesize the available evidence examining the potential role of counselling or PA programs on GDM prevention in high risk populations [15]. In terms of more recent studies, we were able to identify eight (references in supplementary material B) syntheses published during the period between 2012 and 2019 but they all mix up counselling and PA program interventions and thus despite the volume of syntheses, this area remains inconclusive and had a number of methodological issues. For example a 2019 meta-analysis reported findings that are not plausible since they report that nutrition or PA counselling given alone had a significant effect but not the combination of nutrition and PA counselling [16]. They also mix up counselling with PA programs as well as of studies of women at high and low risk and this added to the contradictory results. In addition they included activities like Yoga [17] which is a lower intensity exercise program compared to resistance training. Insufficient consideration of the target population (women at high risk) and type of intervention (counseling vs PA programs) results in an inappropriate classification of studies. The most recent synthesis of experimental studies published in 2019 [18] included 10 RCTs related to the nutrition counselling and exercise intervention of which two were not deemed appropriate to be included $[14,19]$ and three other studies that satisfy the inclusion criteria were not included [20-22]. The authors Inappropriately use a network meta-analysis approach which is not ideal as it is unlikely that any patient in the network could have been given any of the treatments in the network (metformin. probiotics, exercise, vitamin D). As such, the indirect comparisons are not protected by randomisation and are more likely to be confounded by differences between the trials. Similarly, the third 2019 metaanalysis [23] included only five out of eleven potentially rele- vant trials on exercise and thus did not satisfy the inclusion criteria. Thus, despite the proliferation of both RCTs and meta-analyses, the question regarding the efficacy of these PA program interventions remains unresolved and further trials are being planned (such as the PLEDGE trial in Qatar; NPRP10-0213-170456).

Our clinical question for this study is the efficacy of PA intervention programs in women at high risk delivered in pregnancy for GDM prevention. We were commissioned to undertake this synthesis and bring together specialists in relevant areas, our aim being to provide some closure on the uncertainty around the preventative efficacy of PA programs delivered in pregnancy.

\section{Methods}

\subsection{Database search}

Several databases were searched (PubMed, Embase, Cochrane Central Register of Controlled Trials, Cochrane library, ClinicalTrials.gov, WHO website, PsycINFO, and the grey literature (www.opengrey.eu/)). We deliberately conducted a broad search using keywords and synonyms to achieve good coverage. This was followed by a manual search of the top 50 similar citations (PubMed) on studies that were finally included after full text review. Simple structured Boolean searches were also conducted to assess the completeness of our RCT evidence base [24]. A final hand search of references from the selected studies and relevant syntheses was then done to ensure that no relevant RCTs were missed. We report our search strategy in details in supplementary material, section A.

\subsection{Study selection}

We restricted inclusions to human studies after 1966 and till June 2020. Further restrictions were the English language. Selection was also restricted to the RCT design that included the interventions of interest delivered from early pregnancy) as information on incident GDM during pregnancy. Exclusions were made for studies that included low GDM risk participants, had participants with pre-existing diabetes or GDM diagnosed at entry, or were not carried out in routine care settings. Studies were considered to have recruited high-risk participants if they had any of the following:

(a) overweight or obesity

(b) prior GDM or macrosomia

(c) high risk status on a GDM risk assessment tool [25].

No exclusions were made based on diagnosis of GDM according to different international definitions [26-32]

We excluded trials that did not report PA intervention programs, did not compare the intervention with the usual standard of care, were not RCTs or where GDM was not an outcome (primary or secondary). We also pre-specified exclusion of low intensity PA programs such as Yoga and meditation exercises because there is evidence of dose response, with higher exercise intensities producing greater benefits on insulin sensitivity. All studies considered in this analysis excluded 
pre-existing diabetes and GDM diagnosed at entry. This synthesis therefore includes incident GDM data assessed around 24-32 weeks of gestation in women without GDM at entry.

\subsection{Data extraction and quality assessment}

Independent screening of titles, abstracts and full texts by two authors (OM, AM) was carried out. All final decisions were discussed with a third author (SD) when there were controversies and resolved through discussion. A similar approach was used for risk of bias assessment or when data was extracted (e.g. numbers of events). When studies did not report important data or there was an inconsistency, we emailed the corresponding authors. Only two arms were extracted per study and in studies with three or more arms, extraction of data was for the most relevant intervention arm and the control arm. Some studies were reported stratified by BMI which allowed us to include the high risk stratum as these included women at high risk.

We applied the Cochrane Risk of Bias 2.0 tool.[33] for assessment of the quality of the original studies and this has six domains to be assessed (supplementary section D). This tool is used to assess the studies against several bias safeguards across six domains. The domains are then judged at low or high risk of bias in each study and these were counted ( $1=$ high; $0=$ low and $0.5=$ uncertain). A previous study has delineated how such counts may be used to create a relative quality rank (relative to the best study in the metaanalysis) which rescales these counts between 0 and 1 [34]. The latter rank was automatically computed by our software and used for bias adjustment in the meta-analysis $[35,36]$.

\subsection{Data synthesis}

The risk ratio (RR) was the effect size in this synthesis. Overall and pre-specified subgroup analyses were undertaken. The pre-specified subgroups were in-facility supervised exercise or not, recruitment time (before 16 weeks gestation or up to 20 weeks), risk status (dichotomised into overweight/obesity and other high risk criteria) and incidence of GDM in the control arms ( $<=20 \%$ versus $>20 \%$ ). These were undertaken only if there were more than two RCTs per group. We tested for interactions by using a Q-test under fixed effect weights to assess the dispersion of the respective summary effects about the combined effect [37]. This is equivalent to using a Q-test to partition the variance and test the between-subgroups portion of the variance or use a Z-test to compare the two effect sizes directly.

Meta-analysis utilised the quality effects model $[35,36]$ as it is more robust [38] than the classic fixed- or randomeffects models when analyzing heterogeneous studies. We depicted the synthesized data using forest plots and sensitivity to model selection was tested by conducting the analysis using two other models (the robust IVhet model [39] and the conventional random effects model [40] and these are reported in the supplementary material).

We checked for inconsistency using the $\mathrm{I}^{2}$ statistic [41] and checked for possible small study effects through generation of Doi plots and the LFK index [42]. These methods are more reliable [42] than commonly used funnel plots or Egger's regression [43]. Interpretation of these results followed previous guidelines [42]. Only exact P values were reported. All analyses were conducted using Stata version 15 (College Station, TX, USA) and the admetan package [44] and reporting followed the PRISMA [45] guidelines.

\section{Results}

\subsection{Identified studies}

A total of 1599 potentially relevant papers were found across databases and were sorted and accessed using EndNote X7. To this we added additional searches as described in the methods section. After screening of titles and abstracts, and removal of duplicates across the various databases, full texts of 195 papers were retrieved. We excluded studies of specific types of dietary supplements, weight monitoring, meditation type exercises or that did not use usual care controls. Two quasi-experimental trials using convenience sampling were also excludedOther exclusions included women without high risk status and trials with interventions that were nutrition / PA counseling only. All references to exclude trials are given in the supplementary material section B. In all, 11 RCT's (Table 1) finally met the inclusion criteria for this synthesis [20-22,46-53]. The study selection process is depicted in Fig. 1.

\subsection{Study characteristics}

High risk was defined as obesity $[46,50,53]$ or overweight/obesity $[22,47,48,51,52]$ or through the presence of one of several risk factors in addition to or without overweight/obesity $[20,21,49]$ (including prior GDM, prior macrosomia, a risk tool score or age $>35-40 y)$. Interestingly, only the tool used a definition of high risk that considered a family history of type 2 diabetes perhaps because it is not an independent predictor of GDM [54]. While the criteria for high risk vary, they all lead to women whose risk status is greater than the average woman in pregnancy with none of the risk factors thus ensuring that this study included women who were at higher than average risk. All trials were single centre RCTs.

\subsection{Origin of the studies}

One RCT was from Asia [51], three were from Oceania $[20,46,52]$ three were from Europe $[47,49,50]$ and four were from North America [21,22,48,53].

\subsection{Interventions}

Of the 11 RCTs, five $[22,47,49,51,53]$ utilised a health care facility based supervised exercise program while six $[20,21,46,48,50,52]$ were supervised outside of the health care facility. The recruitment was reported to have been prior to the 16 th gestational week in seven $[20,21,46,48,50,51,53]$ studies and up to 20 weeks in four $[22,47,49,52]$ studies. Across the RCTs, intervention continued till approximately 36 weeks and all studies provided participants with pregnancy-specific dietary advice as per local guidelines. Further details of these interventions are given in supplementary material, section $B$. 
Table 1 - Population characteristics of included studies.

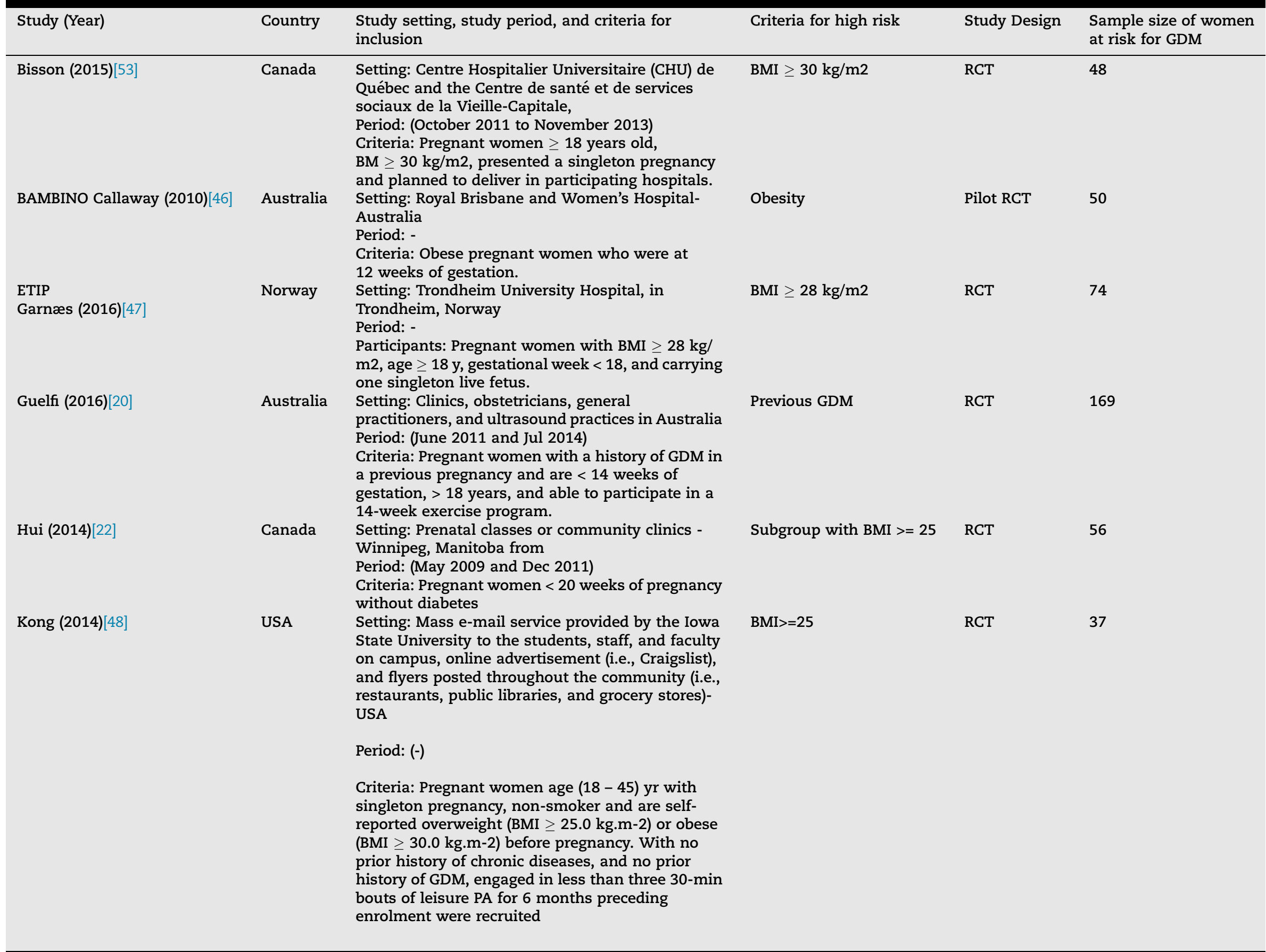




\begin{tabular}{|c|c|c|c|c|c|}
\hline Study (Year) & Country & $\begin{array}{l}\text { Study setting, study period, and criteria for } \\
\text { inclusion }\end{array}$ & Criteria for high risk & Study Design & $\begin{array}{l}\text { Sample size of women } \\
\text { at risk for GDM }\end{array}$ \\
\hline Nobles (2015)[21] & USA & $\begin{array}{l}\text { Setting: Ambulatory obstetrical practices of } \\
\text { Baystate Medical Center-USA } \\
\text { Period: (2007 to 2012) } \\
\text { Criteria: Women in their first trimester of } \\
\text { pregnancy, between the ages of } 16 \text { and } 40 \text {, and at } \\
\text { increased risk for GDM }\end{array}$ & $\begin{array}{l}\text {-BMI }>=25 \\
\text { or Family history of diabetes } \\
\text { or prior GDM according to ADA }\end{array}$ & RCT & 251 \\
\hline Oostdam (2012)[49] & Netherland & $\begin{array}{l}\text { Setting: Hospitals and midwifery practices- } \\
\text { Netherland between } \\
\text { Period: ( } 2007 \text { to 2011). } \\
\text { Criteria: Pregnant women who were overweight or } \\
\text { obese and at risk for GDM, }\end{array}$ & $\begin{array}{l}- \text { BMI }>=25 \\
\text { Or History of macrosomia } \\
\text { Or History of GDM } \\
\text { Or First-degree } \\
\text { relative with T2DM. }\end{array}$ & RCT & 184 \\
\hline $\begin{array}{l}\text { TOP } \\
\text { Renault (2014)[50] }\end{array}$ & Netherland & $\begin{array}{l}\text { Setting: Hvidovre Hospital, University of } \\
\text { Copenhage-Netherlands from } \\
\text { Period: (March } 2009 \text { to March 2012) } \\
\text { Criteria: Obese pregnant women with BMI } \geq 30 \mathrm{~kg} \text { / } \\
\text { m2 }\end{array}$ & $\mathrm{BMI} \geq 30 \mathrm{~kg} / \mathrm{m} 2$ & RCT & 259 \\
\hline Seneviratne (2016)[52] & & $\begin{array}{l}\text { Setting: Home-based intervention, Auckland, New } \\
\text { Zealand } \\
\text { Period: (March } 2013 \text { and Oct 2014) } \\
\text { Criteria: Participants were women aged 18- } \\
40 \text { years, BMI } \geq 25 \mathrm{~kg} / \mathrm{m} 2 \text { and a singleton } \\
\text { pregnancy }<20 \text { weeks of gestation. }\end{array}$ & $\mathrm{BMI} \geq 25 \mathrm{~kg} / \mathrm{m} 2$ & RCT & 74 \\
\hline Wang (2017)[51] & China & $\begin{array}{l}\text { Setting: Peking University First Hospital-China } \\
\text { Period: (Dec } 2014 \text { through July } 2016 \text { ) } \\
\text { Criteria: Nonsmoking women age }>18 \text { years with a } \\
\text { singleton pregnancy who met the criteria for } \\
\text { overweight/obese status BMI } \geq(24-28 \mathrm{~kg} / \mathrm{m} 2) \text { and } \\
\text { had an uncomplicated pregnancy at }<12 \text { weeks }\end{array}$ & $\mathrm{BMI}>=24$ & RCT & 265 \\
\hline
\end{tabular}




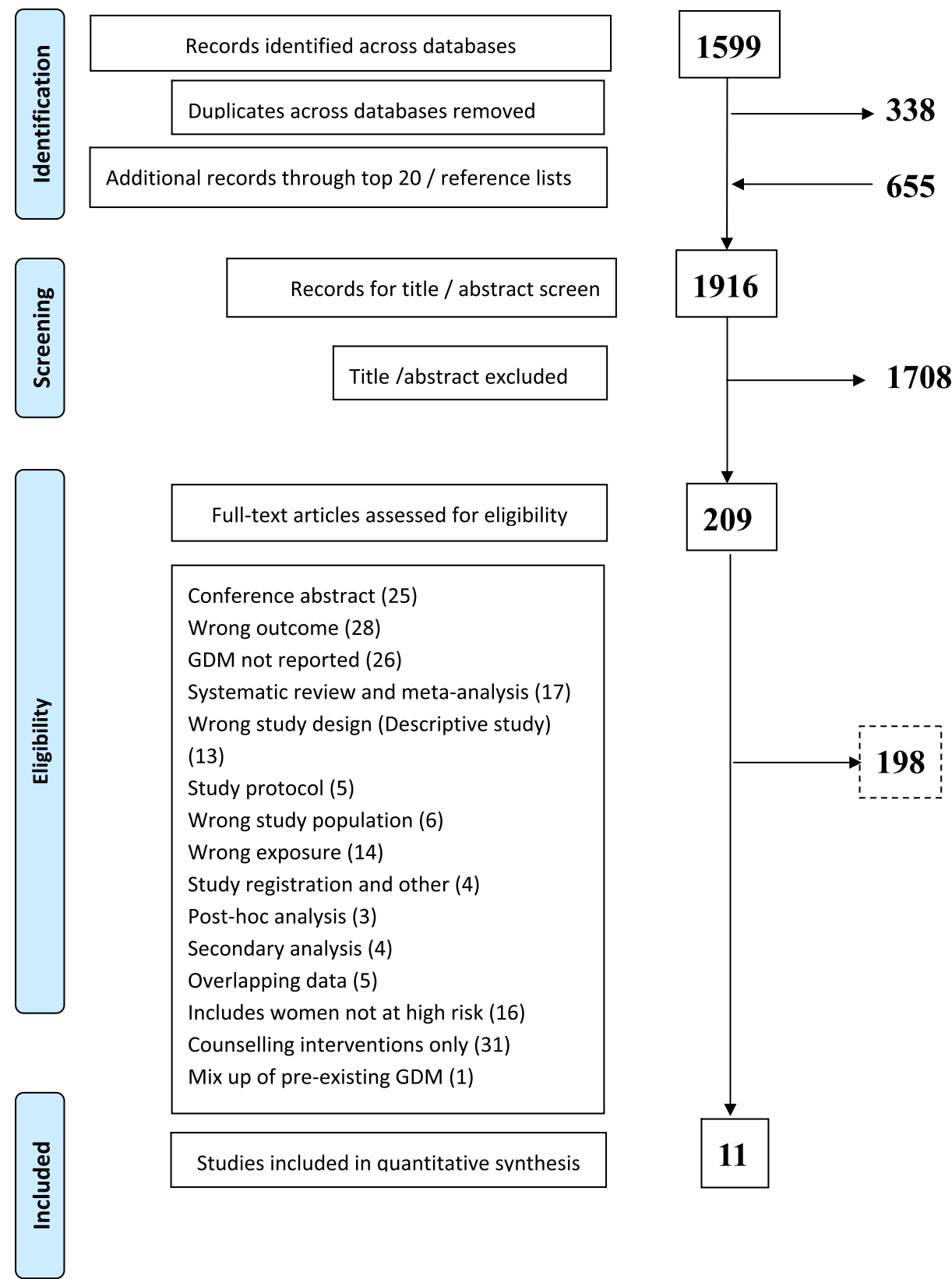

Fig. 1 - Flowchart of the selection of studies.

\subsection{Ascertainment of GDM status}

Seven $[20-22,46,47,51,52]$ studies used the $75 \mathrm{~g}$ glucose tolerance test (GTT) and four [48-50,53] RCTs did not report the glucose dose used for GDM diagnosis. We did not contact the authors to ascertain this information as it was unlikely to impact on these results but was taken into account during quality assessment. Seven GTT diagnostic thresholds were used across studies (supplementary material, section C). The usual timing of the GTT was used and cut-off thresholds are reported in supplementary materials, section C.

\subsection{GDM incidence}

The control group incidence of GDM across the different trials was assessed and ranged between 5 and 41\%. Seven $[21,22,46,48-50,52]$ trials reported under $20 \%$ cumulative incidence (5-15\%) of GDM, two [47,53] reported 21-25\% GDM and the last two [20,51] reported 40-41\% GDM. The latter two studies had high GDM rates because they either limited recruitment to women with prior GDM [20] or had significant attrition [51] perhaps leading to selection bias. 


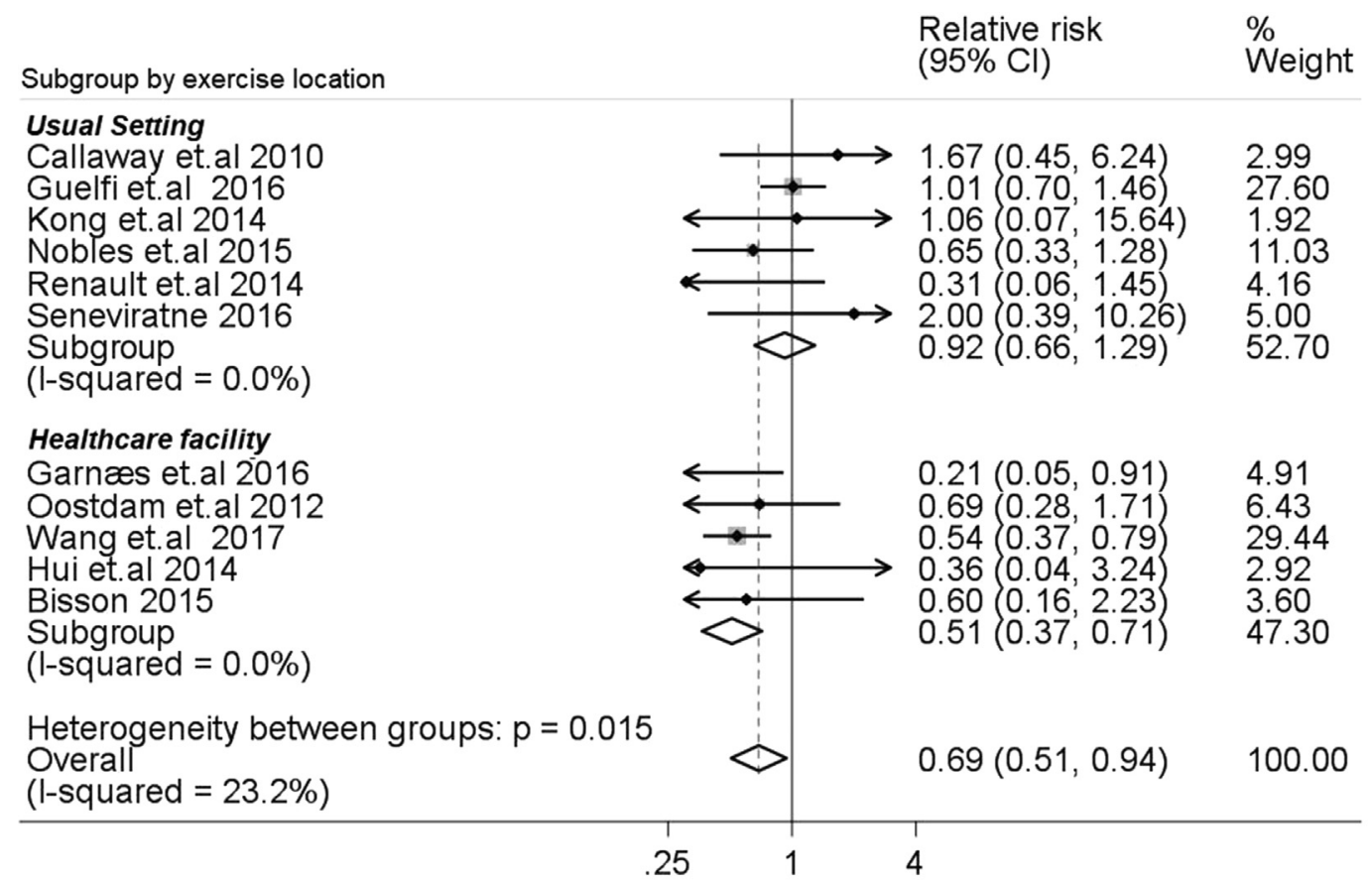

Fig. 2 - Meta-analysis of eleven RCTs of PA program interventions in pregnancy with individual and summary risk ratio's indicated. Weights are from Doi's quality effects model.

\subsection{Quality of the studies}

The least deficient domains (across studies) were attrition, analysis and randomization. The most deficient domain was intervention adherence. Details of the quality assessment for each study is depicted in supplementary material section D. The relative ranks across the studies was between 0.5 and 1 for the 11 RCTs Details are given in supplementary material section D.

3.8. Quantitative synthesis: Magnitude and precision of overall effect

Across 11 trials and 1467 women, 722 received PA interventions through pregnancy and 745 received usual care. GDM developed in 100 PA treated women and in 148 usual care controls (Fig. 2). PA reduced the risk of GDM compared with usual care (RR 0.69, 95\% CI 0.51 to 0.94; Fig. 2). The pooled effect across in-facility exercise was RR 0.51 (95\% CI 0.37 to 0.71 ; Fig. 2). Assuming the $12 \%$ median baseline risk of GDM in women at high risk, this translates to one less GDM occurrence for every 18 (95\%CI 14-29) women undergoing an in-facility PA program in pregnancy compared with usual care.

There was interaction by use of an in-facility exercise venue $(P=0.015$; Table 2$)$. Sensitivity analysis replacing the synthesis model with the IVhet model (RR 0.72; 95\% CI 0.52 to 0.99 ) or with the random effects model (RR 0.70; 95\% CI 0.52 to 0.95 ) produced similar results. The primary results therefore are robust to the selection of the analytic approach even though the three models assign different weights across studies (supplementary material, section E).

\section{Table 2 - Results of pre-specified subgroup analyses.}

\begin{tabular}{|c|c|c|c|c|c|}
\hline Analysis and variable & Studies (n) & $\mathrm{I}^{2}$ & Participants $\left(\mathrm{N}_{\mathrm{PA}}+\mathrm{N}_{\mathrm{con}}\right)$ & Risk ratio $(95 \% \mathrm{CI})$ & Test for interaction $p$-value \\
\hline \multicolumn{6}{|c|}{ In-facility exercise program } \\
\hline Yes & 5 & $0 \%$ & $309+318$ & $0.51(0.37-0.71)$ & \multirow[t]{2}{*}{0.015} \\
\hline No & 6 & $0 \%$ & $413+427$ & $0.92(0.66-1.29)$ & \\
\hline \multicolumn{6}{|l|}{ Incidence of GDM } \\
\hline$<=20 \%$ & 7 & $0 \%$ & $444+467$ & $0.74(0.47-1.17)$ & \multirow[t]{2}{*}{0.736} \\
\hline$>20 \%$ & 4 & 63.6 & $278+278$ & $0.65(0.38-1.13)$ & \\
\hline \multicolumn{6}{|c|}{ Approximate start of trial } \\
\hline Before 16 weeks & 7 & $28.4 \%$ & $532+547$ & $0.71(0.51-1.00)$ & \multirow[t]{3}{*}{0.756} \\
\hline Up to 20 weeks & 4 & $31.2 \%$ & $190+198$ & $0.62(0.26-1.47)$ & \\
\hline \multicolumn{5}{|l|}{ Criterion for high risk } & \\
\hline Other & 3 & $0 \%$ & $296+308$ & $0.87(0.64-1.18)$ & \multirow[t]{2}{*}{0.111} \\
\hline Overweight/Obese & 8 & $8.5 \%$ & $426+437$ & $0.57(0.38-0.86)$ & \\
\hline
\end{tabular}




\subsection{Consistency, directness and publication bias}

The patients, intervention tested and outcome examined were similar to those of interest to clinicians and the healthcare system. Incident GDM in an index pregnancy is certainly of interest to patients and care-givers. All comparisons were head-to-head and these findings are directly of relevance.

Across the 11 RCTs the $\mathrm{I}^{2}$ was $0 \%$ within facility subgroups and thus there was consistency of the effect seen. A cumulative analysis (not shown) suggested that the Garnaes et al trial in 2016, was the point at which there was significance of the results and this was maintained thereafter as additional trials were added on.

The 11 RCTs demonstrated no asymmetry overall of the study effects (supplementary material, section F, left panel; LFK index 0.14). This suggests the absence of small study effects. The funnel plot (supplementary material, section F, right panel) was not clearly interpretable, as expected [55] while Egger's $P(P=0.69)$ also suggested symmetry.

\section{Discussion}

This meta-analysis confirms, for the first time, that PA provided in-facility and started prior to the 16th-20th week of gestation, can prevent emerging GDM in pregnant women at high-risk. This finding was consistent across studies regardless of the different definitions of high risk patients or of the timing of PA initiation.

GDM occurs when pancreatic beta-cells cannot cope with the insulin resistance of pregnancy, and this could be multifactorial including impaired beta cell number, mass or function or a mixture of all these factors [9]. The impact on beta-cells of PA interventions, like that of metformin, must be largely compensatory (addressing insulin resistance) with little opportunity for improvement in beta-cell function over the short period of gestation. Insulin resistance increases later in pregnancy [56], triggering glycaemic deterioration and this is a reflection of pre-existing beta-cell compromise. While the PA effect is sufficient to mitigate GDM related dysglycemia, perhaps by mitigating the increasing insulin resistance expected as pregnancy proceeds, this appears not to be the case with counseling interventions [11-14]. We know that insulin resistance increases during gestation and this increase is larger when GDM ensues [57] and thus PA must have a mitigating effect here. This needs to be explored in future studies.

This synthesis has several strengths over those previously conducted. Eligibility criteria were explicit, the search was very comprehensive and conducted by a specialist librarian, risk of bias assessment was used for bias adjustment, and there was an explicit consideration of reporting bias, subgroup effects and reporting of the GRADE domains in relation to the outcome [58]. A limitation of this study is that we did not register it on PROSPERO although doing so may also have disadvantages such as deterrence of others from similar research that may be of higher quality and other issues raised previously [59]. Another limitation is that we do not know how much the control groups changed after the start of the trials. Contamination of the control group is a real risk with the sort of RCTs we included in this synthesis and could be a factor in the seeming lack of efficacy of these interventions. This would be more of a problem with counseling compared to PA interventions. However there have been two cluster randomised trials (FeLIPO [60] and GeliS [61]) that would have avoided contamination yet demonstrated no efficacy for the counseling intervention in terms of GDM prevention. Both the latter trials included low risk women however. Another limitation of this synthesis is the use of studies where the diagnostic thresholds for GDM varied and we know that certain GTT thresholds yield more cases than others [62,63] This is not a major problem given that comparisons are made within trials and it is unlikely that the intervention modifies the operating characteristics of the specific criterion used to diagnose GDM. Finally, we did not conduct subgroups according to BMI, history of prior GDM or macrosomia as these data were not consistently reported across studies.

We conclude that these results should be brought to the attention of clinicians so that they are aware that PA is of benefit for GDM prevention. Clinicians now have data that supports supervised exercise in pregnancy for the GDM prevention indication. What may now be important for future research is to compare PA in-facility versus outside the health care facility to establish the determinants of its success in GDM prevention. In addition, the possibilities for these interventions are changing a lot with the wealth of mobile and other apps and web pages coming up. Telemedicine support from health centres needs a separate investigation as there might be an opening here that could be more effective. The efficacy of the intervention must also be looked at in the light of patient activation - a measure of patient "quality".

\section{Funding}

This work was made possible by Program Grant \#NPRP100129-170274 from the Qatar National Research Fund (a member of Qatar Foundation). The findings herein reflect the work, and are solely the responsibility of the authors. All authors had full access to all the data in the study and the corresponding author had final responsibility for the decision to submit for publication. LFK was supported by an Australian National Health and Medical Research Council Fellowship (APP1158469)

\section{Declaration of Competing Interest}

The authors declare that they have no conflict of interest.

\section{Acknowledgements}

SAD was responsible for study conception, provided statistical and epidemiological oversight and is guarantor of the article. LT, LFK, JC, OM and AM contributed to the data search, collection and discussion, data extraction and quality assessment. All authors critically reviewed the manuscript for scientific content. ET and SD critically reviewed the manuscript for clinical applicability and relevance. All authors approved the final manuscript. 


\section{Appendix A. Supplementary material}

Supplementary data to this article can be found online at https://doi.org/10.1016/j.diabres.2020.108371.

\section{R E F E R E N C E S}

[1] Callaway LK, McIntyre HD, Barrett HL, et al. Probiotics for the prevention of gestational diabetes mellitus in overweight and obese women: findings from the spring double-blind randomized controlled trial. Diabetes Care 2019;42(3):364-71.

[2] Lee KW, Ching SM, Ramachandran V, et al. Prevalence and risk factors of gestational diabetes mellitus in Asia: a systematic review and meta-analysis. BMC Pregnancy Childbirth 2018;18(1):494.

[3] Bashir M, Baagar K, Naem E, et al. Pregnancy outcomes of early detected gestational diabetes: a retrospective comparison cohort study, Qatar. BMJ Open 2019;9(2) e023612.

[4] Boomsma CM, Eijkemans MJ, Hughes EG, et al. A metaanalysis of pregnancy outcomes in women with polycystic ovary syndrome. Hum Reprod Update 2006;12(6):673-83.

[5] Huvinen E, Eriksson JG, Stach-Lempinen B, Tiitinen A, Koivusalo SB. Heterogeneity of gestational diabetes (GDM) and challenges in developing a GDM risk score. Acta Diabetol 2018;55(12):1251-9.

[6] Kampmann FB, Thuesen ACB, Hjort L, et al. Exposure to gestational diabetes is a stronger predictor of dysmetabolic traits in children than size at birth. J Clin Endocrinol Metab 2018.

[7] Egan AM, Simmons D. Lessons learned from lifestyle prevention trials in gestational diabetes mellitus. Diabet Med 2019;36(2):142-50.

[8] Doi SA, Furuya-Kanamori L, Toft E et al. Metformin in pregnancy to avert gestational diabetes in high-risk women: meta-analysis of randomised controlled trials. Obes Rev 2019; First published: 31 October 2019.

[9] Plows JF, Stanley JL, Baker PN, Reynolds CM, Vickers MH. The pathophysiology of gestational diabetes mellitus. Int J Mol Sci 2018;19(11).

[10] Polley BA, Wing RR, Sims CJ. Randomized controlled trial to prevent excessive weight gain in pregnant women. Int J Obes Relat Metab Disord 2002;26(11):1494-502.

[11] Dodd JM, Turnbull D, McPhee AJ et al. Antenatal lifestyle advice for women who are overweight or obese: LIMIT randomised trial. BMJ 2014; 348:g1285. Notes: CORPORATE NAME: LIMIT Randomised Trial Group.

[12] Poston L, Bell R, Croker H et al. Effect of a behavioural intervention in obese pregnant women (the UPBEAT study): a multicentre, randomised controlled trial. Lancet Diabetes Endocrinol 2015; 3(10):767-77. Notes: CORPORATE NAME: UPBEAT Trial Consortium.

[13] Koivusalo SB, Rono K, Klemetti MM, et al. Gestational diabetes mellitus can be prevented by lifestyle intervention: the finnish gestational diabetes Prevention Study (RADIEL): a randomized controlled trial. Diabetes Care 2016;39(1):24-30.

[14] Simmons D, Devlieger R, van Assche A, et al. Effect of physical activity and/or healthy eating on GDM risk: the DALI lifestyle study. J Clin Endocrinol Metab 2017;102(3):903-13.

[15] Shepherd E, Gomersall JC, Tieu J et al. Combined diet and exercise interventions for preventing gestational diabetes mellitus. Cochrane Database Syst Rev 2017; 11:CD010443.

[16] Guo XY, Shu J, Fu XH, et al. Improving the effectiveness of lifestyle interventions for gestational diabetes prevention: a meta-analysis and meta-regression. BJOG 2019;126(3):311-20.
[17] Rakhshani A, Nagarathna R, Mhaskar R, et al. The effects of yoga in prevention of pregnancy complications in high-risk pregnancies: a randomized controlled trial. Prev Med 2012;55 (4):333-40.

[18] Chatzakis C, Goulis DG, Mareti E et al. Prevention of gestational diabetes mellitus in overweight or obese pregnant women: A network meta-analysis. Diabetes Res Clin Pract 2019;107924.

[19] Daly N, Farren M, McKeating A, et al. A medically supervised pregnancy exercise intervention in obese women: a randomized controlled trial. Obstet Gynecol 2017;130 (5):1001-10.

[20] Guelfi KJ, Ong MJ, Crisp NA, et al. Regular Exercise to Prevent the Recurrence of Gestational Diabetes Mellitus: A Randomized Controlled Trial. Obstet Gynecol 2016;128 (4):819-27.

[21] Nobles C, Marcus BH, Stanek 3rd EJ, et al. Effect of an exercise intervention on gestational diabetes mellitus: a randomized controlled trial. Obstet Gynecol 2015;125(5):1195-204.

[22] Hui AL, Back L, Ludwig S, et al. Effects of lifestyle intervention on dietary intake, physical activity level, and gestational weight gain in pregnant women with different pre-pregnancy Body Mass Index in a randomized control trial. BMC Pregnancy Childbirth 2014;14:331.

[23] Nasiri-Amiri F, Sepidarkish M, Shirvani MA, Habibipour P, Tabari NSM. The effect of exercise on the prevention of gestational diabetes in obese and overweight pregnant women: a systematic review and meta-analysis. Diabetol Metab Syndr 2019;11:72.

[24] Waffenschmidt S, Janzen T, Hausner E, Kaiser T. Simple search techniques in PubMed are potentially suitable for evaluating the completeness of systematic reviews. J Clin Epidemiol 2013;66(6):660-5.

[25] Teede HJ, Harrison CL, Teh WT, Paul E, Allan CA. Gestational diabetes: development of an early risk prediction tool to facilitate opportunities for prevention. Aust N Z J Obstet Gynaecol 2011;51(6):499-504.

[26] Metzger BE, Gabbe SG, Persson B et al. International association of diabetes and pregnancy study groups recommendations on the diagnosis and classification of hyperglycemia in pregnancy. Diabetes Care 2010; 33(3):67682. Notes: CORPORATE NAME: International Association of Diabetes and Pregnancy Study Groups Consensus Panel.

[27] World Health Organization. Definition, diagnosis and classification of diabetes mellitus and its complications: report of a WHO consultation. Part 1, Diagnosis and classification of diabetes mellitus. 1999. Geneva: World health organization.

[28] Diagnosis and classification of diabetes mellitus Diabetes Care 2006; 29 Suppl 1:S43-8. Notes: CORPORATE NAME: American Diabetes Association.

[29] Hoffman L, Nolan C, Wilson JD, Oats JJ, Simmons D. Gestational diabetes mellitus-management guidelines. The Australasian Diabetes in Pregnancy Society. Med J Aust 1998;169(2):93-7.

[30] Gestational Diabetes Mellitus. Practice Bulletin No. 137. American College of Obstetricians and Gynecologists. Obstet Gynecol 122, 406-16. 2013.

[31] National Diabetes Data Group. Classification and diagnosis of diabetes mellitus and other categories of glucose intolerance. Diabetes 28[12], 1039-1057. 1979. Am Diabetes Assoc.

[32] O'Sullivan JB, Mahan CM. Criteria for the oral glucose tolerance test in pregnancy. Diabetes 1964;13:278-85.

[33] Higgins JPT, Sterne JAC, Savovic J et al. A revised tool for assessing risk of bias in randomized trials. Chandler J, McKenzie J, Boutron I, Welch V eds. Cochrane Methods. , Issue 10 (Suppl 1). dx.doi.org/10.1002/14651858.CD201601.: Cochrane Database of Systematic Reviews, 2016. 
[34] Stone JC, Glass K, Munn Z, Tugwell P, Doi SAR. Comparison of bias adjustment methods in meta-analysis suggests that quality effects modeling may have less limitations than other approaches. J Clin Epidemiol 2020;117(36-45).

[35] Doi SA, Barendregt JJ, Khan S, Thalib L, Williams GM. Advances in the meta-analysis of heterogeneous clinical trials II: The quality effects model. Contemp Clin Trials 2015; 45(Pt A):123-9.

[36] Stone JC, Glass K, Munn Z, Tugwell P, Doi SAR. Comparison of bias adjustment methods in meta-analysis suggests that quality effects modeling may have less limitations than other approaches. J Clin Epidemiol 2019;117(36-45).

[37] Altman DG, Bland JM. Interaction revisited: the difference between two estimates. BMJ 326[7382], 219. 2003/01/25.

[38] Doi SAR, Furuya-Kanamori L, Thalib L, Barendregt JJ. Metaanalysis in evidence-based healthcare: a paradigm shift away from random effects is overdue. Int J Evid Based Healthc 2017; 15(4):152-160.

[39] Doi SA, Barendregt JJ, Khan S, Thalib L, Williams GM. Advances in the meta-analysis of heterogeneous clinical trials I: The inverse variance heterogeneity model. Contemp Clin Trials 2015; 45(Pt A):130-8.

[40] DerSimonian R, Laird N. Meta-analysis in clinical trials. Control Clin Trials 1986;7(3):177-88.

[41] Higgins JP, Thompson SG, Deeks JJ, Altman DG. Measuring inconsistency in meta-analyses. BMJ 2003;327(7414):557-60.

[42] Furuya-Kanamori L, Barendregt JJ, Doi SAR. A new improved graphical and quantitative method for detecting bias in meta-analysis. Int J Evid Based Healthc 2018; 16 (4):195-203.

[43] Furuya-Kanamori L, Xu C, Lin L, et al. P value-driven methods were underpowered to detect publication bias: analysis of Cochrane review meta-analyses. J Clin Epidemiol 2019;118:86-92.

[44] Fisher D. ADMETAN: Stata module to provide comprehensive meta-analysis. David Fisher, editor. 2018///Nov. Boston College Department of Economics. Statistical Software Components.

[45] Liberati A, Altman DG, Tetzlaff J, et al. The PRISMA statement for reporting systematic reviews and meta-analyses of studies that evaluate healthcare interventions: explanation and elaboration. BMJ 2009/07/21.;339 b2700.

[46] Callaway LK, Colditz PB, Byrne NM et al. Prevention of gestational diabetes: feasibility issues for an exercise intervention in obese pregnant women. Diabetes Care 2010; 33(7):1457-9. Notes: CORPORATE NAME: BAMBINO Group.

[47] Garnaes KK, Morkved S, Salvesen O, Moholdt T. Exercise training and weight gain in obese pregnant women: a randomized controlled trial (ETIP Trial). PLoS Med 2016;13(7) e1002079.

[48] Kong KL, Campbell CG, Foster RC, Peterson AD, LanninghamFoster L. A pilot walking program promotes moderateintensity physical activity during pregnancy. Med Sci Sports Exerc 2014;46(3):462-71.

[49] Oostdam N, van Poppel MN, Wouters MG, et al. No effect of the FitFor2 exercise programme on blood glucose, insulin sensitivity, and birthweight in pregnant women who were overweight and at risk for gestational diabetes: results of a randomised controlled trial. BJOG 2012;119(9):1098-107.

[50] Renault KM, Norgaard K, Nilas L et al. The Treatment of Obese Pregnant Women (TOP) study: a randomized controlled trial of the effect of physical activity intervention assessed by pedometer with or without dietary intervention in obese pregnant women. Am J Obstet Gynecol 2014; 210 (2):134.e1-9.

[51] Wang C, Wei Y, Zhang X, et al. A randomized clinical trial of exercise during pregnancy to prevent gestational diabetes mellitus and improve pregnancy outcome in overweight and obese pregnant women. Am J Obstet Gynecol 2017;216 (4):340-51.

[52] Seneviratne SN, Jiang Y, Derraik J, et al. Effects of antenatal exercise in overweight and obese pregnant women on maternal and perinatal outcomes: a randomised controlled trial. BJOG 2016;123(4):588-97.

[53] Bisson M, Almeras N, Dufresne SS, et al. A 12-week exercise program for pregnant women with obesity to improve physical activity levels: an open randomised preliminary study. PLoS ONE 2015;10(9) e0137742.

[54] Savvidou M, Nelson SM, Makgoba M, et al. First-trimester prediction of gestational diabetes mellitus: examining the potential of combining maternal characteristics and laboratory measures. Diabetes 2010;59(12):3017-22.

[55] Terrin N, Schmid CH, Lau J. In an empirical evaluation of the funnel plot, researchers could not visually identify publication bias. J Clin Epidemiol 2005;58(9):894-901.

[56] Catalano PM. Obesity, insulin resistance, and pregnancy outcome. Reproduction 2010;140(3):365-71.

[57] Endo S, Maeda K, Suto M, et al. Differences in insulin sensitivity in pregnant women with overweight and gestational diabetes mellitus. Gynecol Endocrinol 2006;22 (6):343-9.

[58] Guyatt GH, Oxman AD, Vist GE et al. GRADE: an emerging consensus on rating quality of evidence and strength of recommendations. BMJ 2008; 336(7650):924-6. Notes: CORPORATE NAME: GRADE Working Group.

[59] Waugh N. Misgivings about PROSPERO in solving the problem of duplicate systematic reviews. BMJ 2013;347 f5496.

[60] Rauh K, Gabriel E, Kerschbaum E, et al. Safety and efficacy of a lifestyle intervention for pregnant women to prevent excessive maternal weight gain: a cluster-randomized controlled trial. BMC Pregnancy Childbirth 2013;13:151

[61] Kunath J, Gunther J, Rauh K, et al. Effects of a lifestyle intervention during pregnancy to prevent excessive gestational weight gain in routine care - the clusterrandomised GeliS trial. BMC Med 2019;17(1):5.

[62] Nguyen CL, Lee AH, Minh Pham N, et al. Prevalence and pregnancy outcomes of gestational diabetes mellitus by different international diagnostic criteria: a prospective cohort study in Vietnam. J Matern Fetal Neonatal Med 2019:1-7.

[63] Behboudi-Gandevani S, Amiri M, Bidhendi Yarandi R, Ramezani TF. The impact of diagnostic criteria for gestational diabetes on its prevalence: a systematic review and metaanalysis. Diabetol Metab Syndr 2019;11:11. 